\title{
Roteiros culturais: modelo teórico na abordagem de conteúdos socioculturais no ensino/aprendizagem de línguas estrangeiras
}

\author{
Alba Escalante (ESCALANTE, Alba) \\ Mestre em Lingüística Aplicada pela Universidade de Brasília - UnB \\ albaescalante@gmail.com \\ Enrique Huelva Unternbäumen (UNTERNBÄUMEN, Enrique H.) \\ Professor Doutor da Universidade de Brasília - UnB \\ huelva@gmail.com
}

\section{Resumo}

O termo roteiro cultural vincula-se a uma abordagem utilizada na análise de normas e valores originários da cultura. Essa perspectiva fundamenta-se na idéia de que a forma de falar de um grupo determinado é uma manifestação de um sistema tácito de regras, ou roteiros, que podem ser reconstruídos a partir dos princípios da metalinguagem semântica natural conforme Wierzbicka e Goddard. O objetivo deste artigo é apresentar o modelo teórico dos roteiros culturais na abordagem de conteúdos socioculturais no ensino/aprendizagem de línguas estrangeiras. Fundamentando-nos em Wierzbicka, explicamos e exemplificamos em que consistem os roteiros culturais, fazendo um recorte de uma pesquisa onde foram levantados e comparados alguns traços do roteiro da negação em falantes de língua espanhola de diversas áreas geolectais e em falantes do português do Brasil. Os resultados indicaram que a maioria dos falantes do português concorda que o mais adequado é evitar negar; se comparado ao roteiro da negação dos falantes de espanhol, a negação em português parece apresentar traços contrastantes com os representantes da Espanha e familiaridade com os da América Hispânica. Finalmente, lançamos algumas propostas de aplicação deste modelo teórico para o tratamento dos conteúdos socioculturais no ensino/aprendizagem de língua estrangeira.

Palavras-chaves: língua, cultura, roteiros culturais, ensino/aprendizagem de língua estrangeira (LE).

\begin{abstract}
The term cultural script is associated with an approach used to analyze cultural norms and values. This perspective is founded on the idea that the manner in which a particular group speaks is a manifestation of a tacit system of rules, or scripts, which can be reconstructed from the principles of natural semantic metalanguage, according to Wierzbicka and Goddard. The purpose of this article is to present the theoretical model of cultural scripts in sociocultural content in foreign language teaching/learning. Based on Wierzbicka, cultural scripts are explained and exemplified, as part of a larger study in which negating scripts were observed in Spanish speakers from various geolectal regions and in Portuguese speakers from Brasil. The results showed that the majority of Portuguese speakers agrees that it is more appropriate to avoid negating; when
\end{abstract}


compared to the negating scripts of the Spanish speakers, the negating in Portuguese appears to present scripts that differ from those of the representatives from Spain and that are similar to those from Spanish America. Suggestions are made for applying this theoretical model to treat sociocultural content in foreign language teaching/learning.

Keywords: language, culture, cultural scripts, foreign language teaching/learning.

\section{Introdução}

Entendemos que a comunicação, mesmo que existam boas intenções entre os participantes, e por simples que seja a situação de intercâmbio, não é um processo totalmente transparente. Essa opacidade na situação comunicativa é acentuada quando os participantes pertencem a línguas/culturas diferentes, o que é cada vez mais freqüente num mundo globalizado cujas fronteiras são, para certas finalidades, cada vez mais permeáveis. Nos processos de ensino/aprendizagem esses aspectos são vitais e, por isso, precisamos de mecanismos que ofereçam ao aprendiz o acesso aos aspectos mais profundos que regem as pautas de comunicação. Também, é imprescindível reconhecer que o desafio consiste em desenvolver ferramentas empíricas capazes de atingir o maior número de elementos, já que por trás dos rituais de comunicação não existem somente códigos lingüísticos, mas outros de substrato cultural.

O tema da cultura e seu vínculo com a linguagem vêm sendo pensados desde o século XIX em termos comparativos, com uma forte tendência a privilegiar elementos mais universais ou mais relativos. Essa tradição se instaura já no século XVIII, quando pensadores, como Johann Heder (1744-1803) e Wilhem Von Humboldt (1762-1836), defendiam a idéia de que as pessoas falam diferente porque pensam diferente, e de que pensam diferente porque suas respectivas línguas lhes oferecem instrumentos também diferentes que permitem conceitualizar o mundo.

Um dos momentos mais importantes nas discussões sobre esta relação pode ser localizado na chamada hipótese Sapir-Whorf. Edward Sapir e Benjamin Lee Whorf tentaram demonstrar que as estruturas das línguas influenciam as formas de pensamento e de comportamento dos seus falantes (KRAMSCH, 2001, p. 11-12). Os questionamentos feitos a essas idéias levaram a considerar duas versões da hipótese Sapir-Whorf, uma forte e outra branda.

Na atualidade, a versão mais forte é rejeitada por grande parte da comunidade científica. Ao comentar sobre as implicações de continuar considerando a versão forte, Kövecses (2006, p. 34) ressalta: "se a nossa língua nativa determinasse de forma estrita a maneira de pensar, não poderíamos traduzir de uma a outra língua” e portanto seríamos prisioneiros delas.

Em vez de determinar, a língua influencia o modo como percebemos o mundo. Wierzbicka (1997, p. 2) expressa essa idéia da seguinte forma: "as palavras são artefatos culturais das sociedades, e elas servem para transmitir atitudes sociais e valores culturais". 2 Desta forma, as discussões mais contemporâneas vêm centrando a sua

\footnotetext{
${ }^{1}$ Texto original: If the strong version were true, it would be impossible, or next to impossible to learn a foreign language [...] if our native language strictly determined the way we think, we could not translate from one language to another. Todas as traduções são de nossa autoria.

${ }^{2}$ Texto original: The words are society's cultural artifacts, and they serve as transmitters of social attitudes and cultural values.
} 
atenção no grau e nas conseqüências desta interdependência, permitindo, inclusive, que as comparações sejam feitas já não somente entre diferentes línguas, mas também entre os falantes de uma mesma língua.

Embora se perceba uma evolução nos estudos sobre cultura, os pesquisadores das diferentes áreas tentam buscar uma redução de certos problemas recorrentes como: as aproximações em abstrato; a ênfase exagerada em alguns dos elementos do fenômeno cultural e o conseqüente comprometimento dos outros; e a falta de ferramentas teóricas para evitar o etnocentrismo que alimenta as visões estereotipadas.

Os roteiros culturais são um modelo teórico construído na relação entre a cognição, a linguagem e a cultura, capaz de explicar como agem os diferentes sistemas na construção do significado. Também, são unidades de análise plausíveis para estudarmos a relação entre língua e cultura de forma empírica, não impressionista e livre de etnocentrismo.

Neste trabalho, vamos expor as possibilidades que os roteiros culturais nos oferecem como modelo teórico para a análise de normas e valores provenientes da cultura que, por se manifestarem na linguagem, podem ser reconstruídos a partir dos princípios da metalinguagem semântica natural ${ }^{3}$ (WIERZBICKA, 1994; GODDARD; WIERZBICKA, 1997, 2007). Ilustraremos com exemplos extraídos de diferentes línguas e, para ressaltar a utilidade deste modelo, proporemos algumas dicas de como podem ser utilizados no tratamento de conteúdos socioculturais no ensino/aprendizagem de línguas estrangeiras.

\section{A Metalinguagem Semântica Natural}

A Metalinguagem Semântica Natural (MSN) é um programa de pesquisa desenvolvido ao longo de trinta anos por um grupo encabeçado por Anna Wierzbicka e Cliff Goddard. Os trabalhos sobre MSN baseiam-se na evidência de que, nas línguas, existe uma espécie de termos centrais comuns a todas. Valendo-se do procedimento da paráfrase redutiva como critério central, foram extraídos um conjunto de itens - por volta de 60 - capazes, na sua combinação, de explicar outras palavras ou termos que seriam mais complexos. São os denominados primitivos semânticos (semantics primes), recolhidos na seguinte tabela:

Semantics primes - English exponents

\begin{tabular}{|l|l|}
\hline Substantives & I, YOU, SOMEONE/PERSON, PEOPLE \\
\hline $\begin{array}{l}\text { Relational } \\
\text { substantives }\end{array}$ & SOMETHING, THING, BODY, PART \\
\hline Determiners & THIS, THE SAME, OTHER \\
\hline Quantifiers & ONE, TWO, SOME, ALL, MANY/MUCH \\
\hline Evaluators & GOOD, BAD \\
\hline Descriptors & BIG, SMALL \\
\hline $\begin{array}{l}\text { Mental/experiential } \\
\text { predicates }\end{array}$ & THINK, KNOW, WANT, FEEL, SEE, HEAR \\
\hline Speech & SAY, WORDS, TRUE \\
\hline Actions and events & DO, HAPPEN, MOVE \\
\hline
\end{tabular}

${ }^{3}$ Natural Semantic Metalanguage (NSM). 


\begin{tabular}{|l|l|}
\hline $\begin{array}{l}\text { Existence and } \\
\text { possession }\end{array}$ & THERE IS/EXIST, HAVE \\
\hline Life and death & LIVE, DIE \\
\hline Time & $\begin{array}{l}\text { WHEN/TIME, NOW, BEFORE, AFTER, A } \\
\text { LONG TIME, A SHORT TIME, FOR SOME } \\
\text { TIME, MOMENT. }\end{array}$ \\
\hline $\begin{array}{l}\text { WHERE/PLACE, HERE, ABOVE, BELOW, } \\
\text { FAR, NEAR, SIDE, INSIDE, TOUCH } \\
\text { (CONTACT) }\end{array}$ \\
\hline Logical concept & NOT, MAYBE, CAN, BECAUSE, IF \\
\hline $\begin{array}{l}\text { Intensifier, } \\
\text { augmentor }\end{array}$ & VERY, MORE \\
\hline Similarity & LIKE/WAY \\
\hline
\end{tabular}

A MSN é uma proposta dinâmica que combina teoria, variedade de descrições e testes inter-lingüísticos. Segundo expõe Goddard, ao fazer uma revisão do estado da questão, existe uma clara tendência ao crescimento das pesquisas utilizando a MSN como ferramenta. Sobre o assunto, o autor explica:

Existe uma grande quantidade de elementos a serem descobertos sobre a linguagem - especificações da estrutura semântica do vocabulário e da gramática (léxico-gramática), sobre a estrutura do discurso e as práticas discursivas, sobre a comunicação não verbal, sobre o fenômeno cultural e semiótico. (GODDARD, 2002, p. 315) ${ }^{4}$

Essa tendência vem sendo incorporada de forma cada vez mais abrangente às pesquisas em diversos campos, além de incrementar aplicações práticas no ensino de línguas e na tradução, áreas centrais dentro da Lingüística Aplicada.

Na idéia dos primitivos semânticos, encontramos imbuída a noção de universalidade lingüística/cultural, o que permite uma abordagem comparativa, não somente entre línguas/culturas diversas, mas também como ferramenta empiricamente válida para os estudos comparativos em grupos de falantes da mesma língua. Desta forma contamos com uma base sólida para desenvolver trabalhos suscetíveis de serem colocadas no marco da pesquisa científica.

Uma das virtudes desta perspectiva é a neutralidade cultural que os primitivos semânticos oferecem, elemento-chave que permite evitar o etnocentrismo principalmente quando tentamos explicar aspectos tais como emoções, afetos ou valores próprios de uma cultura. Tais elementos, vistos numa perspectiva alheia, costumam aparecer deformados ou incorretos devido à tendência natural de abordá-los sob um ponto de vista externo. Existem palavras cuja carga cultural é tão acentuada que, ao serem traduzidas ou explicadas em outras línguas utilizando procedimentos comuns, correm o risco de perderem o sentido (GODDARD; WIERZBICKA, 2007, p. 6).

A MSN é uma ferramenta de base para desentranhar as especificidades culturais imbuídas em ações quotidianas. Formas que, como mencionam Goddard e Wierzbicka (1997, p. 235), quando vistas de maneira superficial podem receber descritores do tipo

\footnotetext{
${ }^{4}$ Texto original: There is an enormous amount yet to be discovered about the language - specific semantic structuring of vocabulary and grammar (lexicogrammar), about discourse structure and discourse practices, about non-verbal communications, and panoply of cultural and semiotic phenomena.
} 
mais ou menos direto ou indireto, mais ou menos cordial. Para os autores, além de ser utilizado na análise semântica, a MSN permite formular as "regras culturais conhecidas como roteiros culturais" (WIERZBICKA, 1991, 1994 apud GODDARD; WIERZBICKA, 1997, p. 236).

Em síntese, a MSN pode ser usada como ferramenta metodológica e teórica na formulação dos roteiros culturais, modelo que passaremos a descrever a seguir.

\section{Os roteiros culturais}

Entre os membros dos grupos sociais, existe um espaço compartilhado de regras tácitas necessárias para o entendimento. Tais condutas, valores ou idéias são originados pela experiência direta ou indireta, em forma de práticas sociais, costumes ou normas que perfilam os traços dessa cultura. Mesmo que inconscientemente, os membros desses grupos são portadores, transmissores e até modificadores desses códigos.

Poderíamos, entretanto, pensar que para termos acesso a esses esquemas de ação, já estabelecidos, bastaria um olhar atento e uma descrição detalhada e criteriosa. Embora essa tenha sido uma prática habitual dentro da antropologia cultural, Wierzbicka (1994) afirma que os resultados obtidos nunca foram suficientes para conseguir descompactar de forma apropriada o fenômeno cultural, e descrever ou estabelecer comparações daquelas manifestações que afetam diretamente os estilos de comunicação.

O modelo dos roteiros culturais permite o acesso às nuances dos rituais de interação e às regras que os regem. É uma abordagem utilizada para analisar normas e valores provenientes da cultura, e fundamenta-se na idéia de que o modo de falar de um grupo é uma manifestação de um sistema tácito de regras, ou roteiros culturais que podem ser reconstruídos (WIERZBICKA, 1994).

A autora argumenta que os roteiros culturais são capazes de esclarecer as diferenças culturais "incluindo aquelas que afetam mais diretamente os estilos de comunicação” (WIERZBICKA, 1994, p. 3). Também, do ponto de vista teórico, é importante ressaltar a ruptura com a tradição gerativista que ignorou a riqueza cultural que participa diretamente na produção da linguagem em uso, porque o significado não existe fora da cultura.

Já em 1983, Hall questionava a falta de um método que permitisse entender de maneira profunda e não impressionista os processos implícitos nas manifestações da fala. "Precisamos saber mais sobre como pensam as pessoas de diferentes culturas" (HALL, 1983 apud WIERZBICKA, 1994, p. 2). ${ }^{5}$ Por isso, entendemos que adotar o modelo dos roteiros culturais é um caminho coerente para evitar sermos superficiais nas descrições que podem, com justiça, ser criticadas por seu caráter etnocêntrico.

Os roteiros culturais permitem descrever e comparar atitudes, valores, normas e suposições pertencentes a uma cultura numa perspectiva neutra e simples. Esses elementos emergem intuitivamente em forma de paráfrases auto-explicativas que, no entanto, possuem rigor empírico.

Sobre as virtudes desta abordagem, os autores ressaltam que, além de permitir o acesso às elaborações mentais, isto é, cognitivas, através das evidências lingüísticas, os roteiros culturais proporcionam uma visão qualitativa dos fenômenos. Por isso, um mesmo traço (o silêncio, as desculpas, a insistência) não precisa ser explicado em

${ }^{5}$ Texto original: We need to know more about how people think in different cultures [...]. 
termos de ausência/presença. O importante não é a freqüência de aparição, mas as explicações subjacentes ao ato de fala. Referindo-se aos contrastes, Wierzbicka (1994, p. 4) explica que: "[...] o uso de um ato de fala pode vincular-se às diferenças qualitativas das normas culturais”. 6

Com esta perspectiva, analisamos a cultura a partir de unidades maiores ao lexema, o que também proporciona maior alcance na análise. Nos roteiros culturais, a cultura adquire um aspecto de encenação, de representação, que catalisa e transporta elementos que podem ser estudados como traços culturais. Uma vez identificados, podem ser submetidos à verificação ou a contraste.

Vejamos então como são construídos os roteiros culturais. O seguinte exemplo aborda as atitudes de dois grupos que compartilham a mesma língua - inglês - sobre a expressão das emoções, na base do livro intitulado Black and White Style in Conflict (KOCHMAN'S, 1991, apud WIERZBICKA, 1994, p. 9 -10). ${ }^{7}$

\begin{tabular}{|c|c|}
\hline BLACK STYLE & WHITE STYLE \\
\hline $\begin{array}{l}\text { Quando eu digo alguma coisa } \\
\text { como esta: } \\
\text { "eu penso isso" “eu não penso } \\
\text { isso” } \\
\text { Eu não quero que essa pessoa } \\
\text { pense que eu sinto alguma coisa } \\
\text { sobre isso } \\
\text { [se a pessoa pensa que eu sinto } \\
\text { alguma coisa quando eu digo algo, } \\
\text { pensará que eu não penso bem] }\end{array}$ & $\begin{array}{l}\text { Quando eu digo alguma coisa como } \\
\text { esta: } \\
\text { "eu penso isso" "eu não penso isso" } \\
\text { Eu quero que essa pessoa saiba que } \\
\text { eu sinto alguma coisa sobre isso } \\
\text { [se a pessoa pensa que eu não sinto } \\
\text { algo quando eu digo alguma coisa, } \\
\text { pensará que eu não penso o que } \\
\text { digo] }\end{array}$ \\
\hline
\end{tabular}

Resumindo, no chamado White Style, a expressão da emoção compromete as idéias, enquanto que para o Black Style, as idéias deverão estar apoiadas na emoção para terem o valor esperado. Um olhar detalhado permite verificar que:

- Parte-se de uma mesma língua (inglês) para expressar coisas diferentes;

- O expressado possui um substrato cultural;

- A caracterização não é dicotômica: racional/emocional;

- O dado de análise é o verbatum;

- Na verbalização estão impressas as razões da supressão ou expressão da emoção;

- As razões da supressão ou manifestação da emoção são um traço característico distintivo entre os grupos que pode ter uma forte relação com os valores intrínsecos que cada grupo outorga a esse traço.

Nesse exemplo, as idéias medulares sobre a expressão da emoção apresentam caminhos opostos numa mesma comunidade lingüística, dividida a partir de uma variável racial.

\footnotetext{
${ }^{6}$ Texto original: [...] and the use of these different speech acts is linked with qualitatively different cultural norms.

${ }^{7} \mathrm{Na}$ citação do exemplo, decidimos manter as denominações na língua de origem, já que com uma tradução dos termos que denominam os grupos ao português, correríamos o risco de extrair ou desviar elementos culturais imbuídos no interior dessas palavras. Trata-se de um sentido construído num marco cultural, numa sociedade, onde tais termos referem-se a valores distintos dos que se refeririam numa outra língua, num outro espaço cultural.
} 
Numa comparação sobre as expressões das emoções entre a cultura polaca e a anglo-americana, Wierbicka (1991, apud GODDARD; WIERBICKA, 1997, p. 243244) ilustra, via roteiro cultural, a tendência dos polacos à expressão desinibida dos sentimentos, sejam estes bons ou ruins, da seguinte forma:

Eu quero que as pessoas saibam o que eu sinto

Quando eu sinto alguma coisa boa, eu quero dizer alguma coisa

Quando eu sinto alguma coisa ruim, eu quero dizer alguma coisa

Se eu sinto alguma coisa boa quando penso em você, eu quero que você saiba disso

Segundo a autora, esse roteiro reflete uma necessidade cultural dos polacos de expressar, a qualquer custo, o que se sente. Aspecto este que contrasta com a tendência, em grande parte da sociedade anglo-americana, de desencorajar a expressão das emoções de forma geral, com exceção de circunstâncias nas quais seja necessário.

Num estudo de caso descritivo, cujo foco era a discussão sobre unidade/diversidade lingüístico/cultural dos falantes da língua espanhola, Escalante (em andamento) realizou um levantamento dos roteiros para o pedido e a negação. Os participantes, quatro no total, representavam algumas das áreas geolectais da língua espanhola (MORENO, 2000). Dois deles eram espanhóis e os outros dois hispanoamericanos. Proporcionou-se um modelo de interação pedido/negação em forma de diálogo e suas impressões foram levantadas numa entrevista. roteiro:

Sobre o pedido, a interpretação do diálogo permitiu o levantamento do seguinte

\begin{tabular}{|c|c|}
\hline $\begin{array}{l}\text { QUANDO DIGO ALGO } \\
\text { COMO... } \\
\text {... tem um evento na tua cidade } \\
\text { em julho e eu estou convidado. Lá } \\
\text { não conheço ninguém e estou } \\
\text { procurando onde ficar. Será que } \\
\text { você conhece um lugar barato ... } \\
\text { QUERO DIZER... } \\
\text { Posso ficar na tua casa? }\end{array}$ & $\begin{array}{l}\text { “Aunque no lo hace explícito" (S2: } \\
\text { 53) } \\
\text { "ENTRE LÍNEAS se puede leer" } \\
\text { (S3: 92) } \\
\text { "Él LO TIENE que interpretar, } \\
\text { leer entre líneas” (S4: 96) }\end{array}$ \\
\hline
\end{tabular}

O levantamento do roteiro da negação foi o seguinte:

$\begin{aligned} & \text { QUANDO UMA PESSOA PEDE } \\ & \text { PARA FICAR NA MINHA }\end{aligned}$
CASA, EU DIGO ALGO COMO
$\begin{aligned} & \text {... muy dice, no estoy aquí, no estoy para } \\ & \text { Justo nessa data não estarei em } \\ & \text { casa. }\end{aligned}$
$\begin{gathered}\text { QUERO (S3:93-94) } \\ \text { Não quero que fique na minha } \\ \text { casa. }\end{gathered}$
$\quad$


No entanto, as opiniões sobre os roteiros variaram entre os participantes e podese estabelecer uma primeira hipótese sobre as diferenças na maneira como, por força da cultura, eles agem ou consideram que é apropriado agir na hora de realizar um pedido.

Neste sentido, conclui-se que na Espanha as solicitudes indiretas não são consideradas apropriadas. De fato, os participantes explicaram que a proximidade afetiva dos participantes da interação é diretamente proporcional à explicitude com que se faz o pedido. Idéia contrária à expressada pelos participantes de origem hispanoamericana que valoraram de modo positivo os pedidos indiretos por considerar que eram educados. A variação se manteve na interpretação da negação aos pedidos. Os participantes de origem espanhola consideraram que a resposta ao pedido deve ser feita diretamente, seja para dizer sim ou não, enquanto os representantes das zonas geolectais localizadas na América hispânica, expressaram dificuldade para negar pedidos.

Outra observação sobre a negação esteve relacionada com a idéia, já apresentada para o pedido, de que vínculo estreito entre os participantes do intercâmbio requer, no caso da Espanha, que o mesmo seja feito de forma direta. Todos os participantes sublinharam a necessidade de acompanhar uma negativa com uma desculpa ou explicação, embora o representante da área caribenha tenha colocado que a expectativa ante o pedido é a aceitação do mesmo.

Durante a pesquisa, a autora, que é professora de espanhol no Brasil, realizou, com uma turma de alunos adultos de nível intermediário, um exercício para observar como acontece a negação de pedidos em português do Brasil. Apresentamos a seguir algumas das informações recolhidas nessa atividade.

O roteiro cultural obtido para a negação foi o seguinte:

\section{QUANDO ALGUÉM QUE HÁ MUITO TEMPO NÃO VEJO \\ E QUE MORA EM OUTRO LUGAR TELEFONA \\ QUERENDO FICAR NA MINHA CASA, E EU NÃO QUERO QUE FIQUE, EU DIGO ALGO COMO:}

pois é, que pena, acho que não vou estar em casa, se tivesse ligado antes, eu ia adorar ter você na minha casa, seria muito bom nos encontrar, será em outra oportunidade

\section{QUERO DIZER:}

Não quero que fique na minha casa

As discussões dos alunos sobre os efeitos de uma negação proporcionaram informações interessantes. Algumas das verbalizações colhidas foram as seguintes ${ }^{8}$ :

Eu não me sinto bem quando não digo o que sinto, mas muitas vezes faço isso. Eu gosto de sim ou não, mas as pessoas me criticam e tenho muitos problemas. O melhor é dizer as coisas diretamente, mas se disser 'não' a gente precisa se justificar.

\footnotetext{
${ }^{8}$ Cada uma das intervenções corresponde a alunos diferentes. Trata-se de um grupo de adultos, de ambos os sexos, todos com formação superior em diversas áreas.
} 
Podemos ferir, e não é bom ferir as pessoas.

Quando vivia na Espanha me sentia muito incomodada quando as pessoas

falavam 'não', ficava muito triste.

Não querer é uma ofensa.

Às vezes você tem que inventar para não parecer grosso.

Se alguém te liga, você enrola.

Se você quiser preservar a amizade não pode ferir as pessoas.

Este exercício permitiu-nos deduzir alguns traços característicos do roteiro da negação no Brasil. Embora existam apreciações individuais, a maioria concorda que o mais adequado é evitar negar. No caso em que seja imperativa uma negação, esta deve vir seguida de uma manifestação de lamento e/ou de uma justificação. Deste modo, pensamos que o valor imbuído na dificuldade para negar é o temor de magoar aos outros, ou o temor de perda de laços de amizade. Se comparado ao roteiro da negação dos falantes de espanhol, a negação em português parece apresentar traços contrastantes com os representantes da Espanha e familiaridade com os da América Hispânica, embora nestes últimos o valor subjacente à dificuldade da negação esteja, segundo a pesquisa, mais relacionado com uma espécie de sentimento de culpa, de acordo com informação proporcionada pela representante da região caribenha.

Como podemos observar, as normas e valores culturais podem ter diferentes significados sociais em diferentes ambientes culturais e, nesse sentido, também é necessário considerar aspectos como a permeabilidade das fronteiras nos grupos de contato, variáveis como sexo, idade, raça, além das mudanças que essas normas e valores podem sofrer pelo fato de não serem estáticos.

\section{Algumas aplicações no ensino/aprendizagem de línguas estrangeiras}

No processo de ensino/aprendizagem de línguas estrangeiras, o tratamento dos conteúdos socioculturais costuma ser, além de muito atrativo, tanto para alunos quanto para professores, uma oportunidade para elucubrações, principalmente quando o professor não pertence à comunidade cultural da língua meta, já que, mesmo que domine a língua e embora tenha tido contato direto com esse espaço sociocultural, pertence a uma outra comunidade lingüístico/cultural e, portanto, domina os seus próprios códigos culturais. Mesmo que aprender uma língua signifique a apropriação da cultura, é muito ambicioso pensarmos que nesse processo nos transformamos num membro legítimo de uma sociedade onde não fomos criados. O que adquirimos, na melhor das hipóteses, é um bom domínio desses elementos culturais, uma compreensão que, no diálogo com a nossa própria bagagem, constitui o chamado espaço da interculturalidade, no qual os diferentes ingredientes convivem em harmonia.

No caso do chamado "nativo", entendemos que o seu acesso aos conhecimentos culturais é também limitado. Um exemplo evidente pode estar representado por um professor de espanhol que, como sabemos, é uma língua espalhada por um amplo número de países. Os membros desses territórios, embora tenham em comum a língua e alguns traços culturais, pelo simples fato de pertencerem a países diferentes, construídos em conseqüência de processos históricos também diferentes, apresentam traços culturais que os distinguem. Essa diversidade pode incluir aspectos socioculturais, como gênero, idade, raça, e demais ingredientes que nos fazem também seres individuais. Daí que, sem chegar aos extremos da constituição psíquica de cada ser humano, possamos partir de diversos níveis de abstração para analisarmos as manifestações culturais presentes numa ampla gama de manifestações interativas. 
Na consideração de grupos culturais, podemos estabelecer - e é essa a nossa proposta - uma abordagem dos elementos socioculturais a partir do modelo teórico dos roteiros culturais para destilar elementos que, de outra maneira, podem ser imperceptíveis.

Um exemplo de aplicação prática dos roteiros culturais seria uma proposta didática na qual sejam analisadas as amostras de língua que aparecem, em forma de diálogo, nos materiais didáticos. Ao fazer uma análise dos diferentes atos de fala que se manifestam nesses diálogos e ao estabelecer uma leitura dos mesmos, traduzindo-os em roteiros culturais, poderemos ter acesso aos traços culturais que ali se retratam. Essa prática, embora com outros fins, pode ser empreendida também pelos profissionais encarregados da escolha de materiais didáticos, e a adequação desses diálogos às práticas convencionalizadas nos grupos representados ser utilizada como critério de avaliação desses materiais.

Se em lugar de repetir os diálogos que nos proporcionam os materiais didáticos, identificarmos e descrevermos os atos de fala representados, poderemos discutir com os alunos as diferentes manifestações performáticas presentes nos mesmos e estabelecer contrastes entre as formas apresentadas, e a maneira em que a mesma situação aconteceria na língua/cultura do aluno; afinal, é mais rentável e conveniente chamar a atenção sobre o que acontece nesses processos inconscientes que executamos em nosso cotidiano, do que fazer abstrações sobre realidades que nos são alheias. Uma outra atividade consistiria na desconstrução de diálogos de filmes. Com eles, identificam-se as ações em cumprimentos, pedidos, negações, despedidas, etc., elabora-se o roteiro da cultura alvo e este contrasta-se com o correspondente na cultura do aluno.

É importante ressaltar que, embora essas ações sejam parte do que fazemos no dia-a-dia, ao se tratar de aspectos rotineiros, na maioria dos casos não somos cientes de como acontecem em nossa língua/cultura, e por isso o exercício de contraste pode ser muito rico para entendermos como são esses rituais de interação, tanto em uma como em outra língua/cultura. Também podemos ressaltar que essas formas são realizadas para atingir objetivos e criar situações onde, como conseqüência de uma manifestação performática distinta à esperada, aconteça um choque cultural ou mal-entendido entre os participantes e solicitar que eles identifiquem as causas do atrito.

Com estas propostas, estamos sugerindo uma alternativa para deixar de lado o perigoso território das impressões, para dar passo à utilização de um conhecimento que consideramos genuíno na medida em que assumimos ser membros ativos de um grupo sociocultural, e possuímos como bem próprio os traços lingüístico/culturais que nos identificam como membros do mesmo. Somos seres eminentemente semióticos e isso se evidencia nas nossas manifestações verbais e nas condutas. Manifestações estas que possuem uma forte motivação cognitiva e cultural, o que nos permite pensarmos na busca equivalências na nossa língua/cultura de origem para facilitarmos uma melhor compreensão de conteúdos provenientes de outras línguas/culturas.

Este trabalho é apenas a ponta do iceberg. A abordagem dos roteiros culturais é um campo imenso para pesquisas, cujas contribuições para o desenvolvimento da Lingüística Aplicada, e outras áreas afins, são de grande importância. 


\section{Referências}

ESCALANTE, Alba. Roteiros culturais, 'frames' e metáforas conceituais: abordagens para o estudo da unidade/diversidade lingüística/cultural dos falantes da língua espanhola. Dissertação (Mestrado em Lingüística Aplicada) - Departamento de Línguas Estrangeiras e Tradução, Instituto de Letras, Universidade de Brasília, Brasília, em andamento.

GODDARD, Cliff. On-going development of the NSM research program. In:

WIERZBICKA, Anna. (Eds.). Meaning and universal grammar - theory and empirical findings. Amsterdam/Philadelphia: John Benjamins, 2002. v. II. p. 301-314.

GODDARD, Cliff; WIERZBICKA, Anna. Discourse and culture. In: van DIJK, Teun A. (Ed.). Discourse as a social interaction. London: Sage, 1997. p. 231-259.

Semantics primes and cultural scripts in language learning and intercultural communication. In: FARZARD, Sharifian; PALMER, Gary. (Eds.). Applied cultural linguistics: implications for second language learning and intercultural communications. Amsterdam/Philadelphia: John Benjamins, 2007.

MORENO, Francisco. Qué español enseñar. Cuadernos de didáctica del español/LE. Madrid: Arco Libros, 2000.

KRAMSCH, Claire. Language and culture. Oxford: Oxford University Press, 2001.

KÖVECSES, Zoltán. Language, mind and culture. A practical introduction. Oxford: University Press, 2006.

WIERZBICKA, Anna. Cultural Scripts: A semantic approach to cultural analysis and cross-cultural communications. Pragmatics and Language Learning. Monograph Series. v. 5, 1994. p. 2-25.

The double life of a bilingual: a cross-cultural perspective. In: BOND, Michael. (Ed.). Working at the interface of culture: eighteen lives in social sciences. London: Routledge, 1997. p. 113-125. 